www.nature.com/jhg

\title{
REVIEW
}

\section{Genotype-phenotype associations and human eye color}

\author{
Désirée White and Montserrat Rabago-Smith
}

\begin{abstract}
Although eye color is usually modeled as a simple, Mendelian trait, further research and observation has indicated that eye color does not follow the classical paths of inheritance. Eye color phenotypes demonstrate both epistasis and incomplete dominance. Although there are about 16 different genes responsible for eye color, it is mostly attributed to two adjacent genes on chromosome 15, hect domain and RCC1-like domain-containing protein 2 (HERC2) and ocular albinism (that is, oculocutaneous albinism II (OCA2)). An intron in HERC2 contains the promoter region for OCA2, affecting its expression. Therefore, singlenucleotide polymorphisms in either of these two genes have a large role in the eye color of an individual. Furthermore, with all genetic expression, aberration also occurs. Some individuals may express two phenotypes-one in each eye-or a complete lack of pigmentation, ocular albinism. In addition, the evolutionary and population roles of the different expressions are significant. Journal of Human Genetics (2011) 56, 5-7; doi:10.1038/jhg.2010.126; published online 14 October 2010
\end{abstract}

Keywords: eye color; HERC2; OCA2; SNP

\section{INTRODUCTION}

In the most elementary form, the inheritance of eye color is classified as a Mendelian trait. ${ }^{1}$ On the basis of the observation of more than two phenotypes, eye color has a more complex pattern of inheritance. Eye color ranges include varying shades of brown, hazel, green, blue, gray, and in rare cases, violet and red. The traditional view was correct in which an allele that codes for brown is dominant over green or blue, and green takes precedence over blue. ${ }^{2}$

Melanocytes in the stroma and anterior layers of the eye hold melanin in their cytoplasms. In the rest of the body, the melanin is secreted from the cells. This provides an explanation why some babies develop their eye color, but skin pigmentation changes constantly throughout life. Despite the color of the eye, the number of melanocytes does not differ. The quantity and quality of melanin in the cytoplasm determines the observed color of the eye. When light passes through a large amount of melanin, most of the visible light is absorbed, and the little that is reflected back appears brown. This same phenomenon is the reason why the pupil appears black. All visible light is absorbed by the retina. As the eye color lightens, less melanin is present in the cells, reflecting more of the visible spectrum. Red and violet eyes come from a lack of pigment. The red appearance is the reflection of the eye's blood vessels. When there is too little pigment to produce a strong blue color, the red reflections interact with the small amount of blue, producing a violet color. ${ }^{3}$

The biological process for producing melanin, melanogenesis, involves numerous protein interactions. In melanocyte-specific organelles known as melanosomes, two pathways for melanogenesis occur. One leads to eumelanin, a darker pigment (brown-black), and the other to pheomelanin, a light pigment (red-yellow). Tyrosinase (TYR), the enzyme responsible for pigment production in the body, starts the synthesis of both types of melanin by catalyzing a reaction between tyrosine and dopa, forming dopaquinone. In the presence of cysteine, the reaction will proceed to form pheomelanin. To form eumelanin, dopachrome tautomerase, TYR, and TYR-related protein 1 complete the chemical pathway from dopaquinone. ${ }^{3}$

Although the aforementioned proteins are responsible for the production of melanin, once it has been produced in the melanosomes, other proteins are responsible for melanin maturation. Membrane-associated transporter protein and p protein oculocutaneous albinism II (OCA2) transport melanosomes for melanin maturation. Melanocortin 1 receptor (MC1R) instructs a melanocyte to switch production between eumelanin and pheomelanin. ${ }^{3-5}$ Therefore, these two proteins affect the quality and quantity of the melanin in the cell. Other very minor genes are responsible for eye color production, such as agouti signaling protein, but they usually have miniscule effects. ${ }^{5}$

\section{HECT DOMAIN AND RCC1-LIKE DOMAIN-CONTAINING PROTEIN 2 (HERC2) AND OCA2}

Finally, two major genes are responsible for eye color: HERC2 and OCA2. During the first studies to classify genes for eye color, OCA2 was believed to be the dominating factor for eye color determination. ${ }^{3,6-8}$ Within the last couple of years, HERC2, an ubiquitin ligasecoding region, has been linked more strongly to eye color. Both genes are located on chromosome 15. OCA2 ranges from 15q11.2-12 and HERC 2 starts at 15q13. These genes are of the greatest importance for eye color. ${ }^{9-11}$ 
Numerous ubiquitin ligases are coded for throughout the body. Chromosome 15 contains HERC1 and HERC2. Problems with just HERC2 lead to nerve tissue malfunctioning, small size and semisterility or sterility. They help with hormone secretion, which affects the pituitary and can lead to dysfunction of the hypothalamus and other protein complexes. The large HERC2 gene requires $211 \mathrm{~kb}$ and 93 exons that codes for a $528 \mathrm{kDa}$ protein made of 4834 residues. $^{12}$

OCA2 codes for a major transmembrane protein in the melanosome maturation process: $\mathrm{P}$ protein. Similar to membrane-associated transporter protein, it transports melanosomes, but additionally, it controls their $\mathrm{pH} .{ }^{3,13}$ Therefore, the $\mathrm{P}$ protein encoded by OCA2 affects the amount and quality of melanin that deposits in melanocytes. In mice and humans where the $\mathrm{P}$ protein is nonfunctional, albinism occurs, indicating its crucial role in pigmentation. ${ }^{13,14}$ The gene located $11.7 \mathrm{~kb}$ from HERC2 requires $345 \mathrm{~kb}$, but it requires only 24 exons to produce a $110 \mathrm{kDa}$ protein with 838 residues. These two seemingly unrelated genes have a major effect on eye color in humans.

\section{GENOTYPE-PHENOTYPE INTERACTIONS}

The promoter region for OCA2 is located within the HERC2 gene. Although introns are usually viewed as superfluous DNA, intron 86 of HERC2 regulates the expression of OCA2. In studies with HERC2 functions, deletions caused hypopigmentation even though the protein has nothing to do with pigmentation. Further studies of this region and its sequence revealed that a change in one nucleotide, single-nucleotide polymorphism (SNP), regulates the binding site for the transcription of the OCA2 gene, altering its expression. ${ }^{9}$ The base changes from a thymine to a cytosine. The SNP, rs12913832, causes a phenotype change from brown to blue eyes, respectively. In the case of the mutation within HERC2, the expression of the P protein encoded by OCA2 decreases, effectively decreasing its effects in pigmentation. This also explains why deletions within HERC2 would cause a decrease in melanin without interacting with the $\mathrm{P}$ protein itself.

The OCA2 gene also contains numerous regions for eye color expression. Over 300 SNPs for eye color have been identified on the gene, but classification of their results proved too arduous. The gene contains a main coding region for brown eyes (BEY2 15q11-15) and hazel eyes (BEY1)., ${ }^{3,5}$ Other SNPs result in blue and green eyes. One SNP has been studied to show a large significance for eye color. Before the revelation of the effect of HERC2, rs1800407 in exon nine was thought to be the main factor for eye color. The change of this base from a $\mathrm{C}$ to a $\mathrm{T}$ causes a change from brown eyes to non-brown eyes (usually blue). In the $\mathrm{P}$ protein, the mutation causes residue 419 to change from an arginine to a glutamine. The possible changes in the DNA sequence are GCT to GTT and GCC to GTC. Although the crystal structure has not been published for the $\mathrm{P}$ protein coded by OCA2, residue 419 is predicted to face the cytoplasmic portion of the lipid bilayer in one of the several transmembrane domains. ${ }^{14}$ Therefore, the SNP change that results in R419Q most likely affects the $\mathrm{P}$ protein in conformation.

Decreased expression of OCA2 affects the pathway for melanosome maturation. Melanin undergoes a packaging process and if large amounts of $\mathrm{P}$ protein are not available to process and transport it, the quality of the darker pigment is compromised and lighter shades will result. ${ }^{14}$ Demonstrating epistasis, the HERC2 gene affects the results produced by the OCA2 gene. Even if the OCA2 gene contains the alleles for brown eyes, the SNP in intron 86 of HERC2 will prevent its expression. The decreased expression could account for incomplete dominance, as well. Lighter shades of brown and gray, a lighter shade of blue, show a mixture of two phenotypes where neither dominates completely.

\begin{tabular}{|c|c|c|}
\multicolumn{3}{|c}{ hhOo $\times$ Hhoo } \\
\hline & hO & ho \\
\hline Ho & Hh Oo & Hh oo \\
\hline ho & hh Oo & hh oo \\
\hline
\end{tabular}

Figure 1 Blue-eyed Cross.

In the traditional view of eye color, it was taught that two blue-eyed individuals could only produce blue-eyed offspring. With the revelation of this epistatic relationship, it helps to prove that it can, and does, happen. A simple cross is provided in Figure 1: Blue-eyed Cross. ('H' represents the non-mutated HERC2 SNP, and 'O' represents the OCA2 allele for brown eyes). The first parent contains the mutation in the HERC2 intron in both alleles but possesses an allele with the coding for brown eyes. The second parent has a non-mutated HERC2 allele but does not have the coding for brown eyes in the OCA2 gene. More than likely, their offspring would have blue eyes, but a $25 \%$ chance stands that offspring would have brown eyes.

As mentioned previously, melanogenesis produces two different types of melanin and requires numerous proteins. TYR, located from 11q14-q21, begins the melanogenesis pathway. Dopachrome tautomerase (13q32) and TYR-related protein $1(9 \mathrm{p} 23)$ will continue the pathway to form eumelanin. ${ }^{15}$ Therefore, if any of these proteins are not coded for correctly, the eumelanin production may be hindered, producing lighter eyes. In the case of TYR, melanin production will halt entirely, resulting in albinism in the entire body. Although TYR does not code for color, a nonfunctioning TYR masks any other gene responsible for pigmentation.

In the pheomelanin pathway, the presence of cysteine has a major role. Without cysteine, the synthesis cannot be carried out. Although cysteine is not an essential amino acid and its deficiency rarely occurs, the lack of it halts the production of pheomelanin. During pigment distribution in an infant, a diet low in cysteine or methionine, which it is synthesized from, would likely affect the color of the child's eyes until the amino acid is supplemented. In this case, pleiotropic effects change eye color.

Aside from HERC2 and OCA2, the other genes involved in melanin production have some regions that correlate to other eye colors. ${ }^{5}$ MC1R contains regions that increase the probability of obtaining green eyes. This gene is often referred to as 'the red-headed gene' because of its prevalent expression in people with red hair and green eyes. ${ }^{4}$ Dopachrome tautomerase also contains regions for hazel and green eyes. ${ }^{5}$ Regions for brown eyes dominate the effects of these genes, though. Incomplete dominance shows in individuals with lighter shades of brown and hazel. A golden-brown iris indicates the mixture of both eumelanin and pheomelanin (produces the yellow color), and hazel is usually a mixture of brown and green or blue and green, depending on the shade.

A few disorders are associated with eye color. The most common, which the OCA2 gene is named for, is oculocutaneous albinism. An individual with this disorder produces little or no pigment in their ocular melanocytes. This condition is pronounced in people who produce little to no pigment throughout their entire body, but it can be localized to the eyes. ${ }^{2}$ When they produce no pigment at all, it is usually due to a nonfunctioning TYR. ${ }^{10}$ With this condition, a complete lack of pigment produces red eyes, and a small amount of pigment may produce violet eyes. Similar to a lack of TYR, other conditions cause ocular albinism. Disorders in the HERC regions of 
chromosome 15 cause Prader-Willi or Angelman's syndrome. PraderWilli syndrome is inherited from the paternal side whereas Angelman's comes from the maternal side. ${ }^{16-19}$ These syndromes result in hypopigmentation, along with delayed development, seizures and child-like behavior patterns..$^{10,12}$

Heterochromia, although not viewed as a severe disorder, affects many individuals. The disorder is characterized by different-colored irises or different colors within the iris. It is inherited or caused by somatic mutations within the cells. ${ }^{2}$ In addition, it can be caused by the inactivation of particular genes within the cells. As different genes may be transcribed in various cells, certain cells will produce more pigment or a different type of pigment than other adjacent cells. The eumelanin/pheomelanin switch triggered by the $M C 1 R$ gene may account for some cases of this disorder.

On the basis of population studies, scientists speculate that the blue-eyed mutation originated in peoples of Northern Europe (Scandinavian countries). Blue is confined mostly to people who originated from Europe. ${ }^{11}$ Green eyes permeate the lowest amount of the population (excluding the disorders), probably due to the lack of coding for it within the genome. Many more genes affect brown and blue more than the other eye colors. Also, if an allele does code for brown, the individual will have brown eyes, except for cases where a critical gene is turned off. Green eyes require more pigment than blue and not much less than brown, and because the shades of hazel (brown with blue or green) are more versatile, hazel is still more popular than green.

The next steps in eye color research involve hue classification among populations. Already, some researchers have started studying hues and saturations. This information revealed more factors for determining eye color in European populations. ${ }^{20}$ Tully, Valenzuela and Zaumseger suggest using genotype data for forensic analysis. By analyzing the DNA from a crime scene, the general phenotypic traits of the suspect may be pieced together. ${ }^{21-23}$ Tully suggests that it may help eliminate particular groups of suspects in circumstances with few leads. In terms of disease, OCA2 and MC1R were linked to melanoma. Different SNPs on these two genes were investigated and analyzed for melanoma risk. ${ }^{24,25}$

\section{CONCLUSIONS}

Eye color results from varying degrees of melanin produced in the melanocytes of the iris. The process that produces melanin, known as melanogenesis, requires numerous proteins. TYR, the enzyme responsible for pigment throughout the body, uses tyrosine to begin the chemical pathway. With the help of dopachrome tautomerase and TYR-related protein 1, eumelanin, the darker pigment, is synthesized; with cysteine, pheomelanin, a yellow-red pigment, is produced. Once the pigment is produced, MC1R, membrane-associated transporter protein, and p proteins (OCA2) mature the melanosomes to be used in the cells.

Two major genes on chromosome 15 affect the quantity and quality of the melanin produced by melanogenesis. HERC2, a large ubiquitin ligase, contains the promoter region for OCA2, the $\mathrm{P}$ protein. When a $\mathrm{T}$ is replaced with a $\mathrm{C}$ in rs12913832 of intron 86 , OCA2 transcription is depressed, resulting in a blue-eyed individual. This epistatic relationship demonstrates the significance of introns and how a singlebase change greatly affects an aspect of the individual. OCA2 contains regions for the numerous eye colors, but one SNP is a strong predictor for brown/blue eyes. A change in rs1800407 causes a change in the protein, Arg419Gln, and a change from brown to blue eyes. Therefore, the residue change causes a problem with the $\mathrm{P}$ protein, and melanin maturation decreases. Aside from these two genes, the genes involved in melanogenesis and other minor genes also contain regions that account for eye color. Having little effect on eye color, many of them deal primarily with hair and skin pigmentation. Lastly, disorders involved in eye color include ocular albinism and heterochromia. Depending on how little pigment the melanocytes produce, albinism causes red or violet eyes. Producing multicolored irises, heterochromia stems from mutations in certain cells of the iris.

1 Hurst, C. C. On the inheritance of eye colour in man. Molecular and General Genet. MGG 1, 393-394 (1908).

2 Redei, G. Encyclopedia of Genetics, Genomics, Proteomics, and Informatics, 3rd edn (Springer, New York City, NY, 2008)

3 Sturm, R. \& Frudakis, T. Eye Colour: portals into pigmentation genes and ancestry. trends Genet. 20, 327-332 (2004).

4 Branicki, W., Brudnik, U. \& WojasPelc, A. Interactions between HERC2, OCA2, and MC1R may influence human pigmentation phenotype. Annals of Hum Genet 73, 160-170 (2009).

5 Frudakis, T., Thomas, M., Gaskin, Z., Venkateswarlu, K., Chandra, K. S., Ginjupalli, S. et al. Sequences associated with human iris pigmentation. Genetics 165, 2071-2083 (2003).

6 Duffy, D. L., Montgomery, G. W., Chen, W., Zhao, Z., Le, L., James, M. R. et al. A threesingle-nucleotide polymorphism haplotype in intron 1 of OCA2 explains most human eye-color variation. Am J Hum Genet 80, 241-252 (2007).

7 Sulem, P., Gudbjartsson, D., Stacey, S., Helgason, A., Rafnar, T., Magnusson, K. P. et al. Genetic determinants of hair, eye and skin pigmentation in Europeans. Nat Genet. 39, 1443-1452 (2007).

8 Zhu, G., Evans, D., Duffy, D., Montgomery, G., Medland, S., Gillespie, N. A. et al. A genome scan for eye color in 502 twin families: most variation is due to a QTL on chromosome 15q. Twin Res 7, 197-210 (2004).

9 Eiberg, H., Troelsen, J., Nielsen, M., Mikkelsen, A., Mengel-From, J., Kjaer, K. et al. Blue eye color in humans may be caused by a perfectly associated founder mutation in a regulatory element located within the HERC2 gene inhibiting OCA2 expression. Hum Genet 123, 177-187 (2008).

10 Kayser, M., Liu, F., Janssens, A. C., Rivadeneira, F., Lao, O., van Duijn, K. et al. Three genome-wide association studies and a linkage analysis identify HERC2 as a human iris color gene. Am J Hum Genet 82, 411-423 (2008).

11 Sturm, R., Duffy, D., Zhao, Z., Leite, F., Stark, M., Hayward, N. et al. A single SNP in an evolutionary conserved region within intron 86 of the HERC2 gene determines human blue-brown eye Color. Am J Hum Genet 82, 424-431 (2008).

12 Garcia-Gonzalo, F. R. \& Rosa, J. L. The HERC proteins: functional and evolutionary insights. Cell Mol Life Sci 62, 1826-1838 (2005).

13 Brilliant, M. The mouse $\mathrm{p}$ (pink-eyed dilution) and human $\mathrm{P}$ genes, ocular albinism type 2 (OCA2), and melanosomal pH. Pigment Cell Res 14, 86-93 (2001).

14 Rinchik, E. M., Bultman, S. J., Horsthemke, B., Lee, S., Strunk, K. M., Spritz, R. A. et al. A gene for the mouse pink-eyed dilution locus and for human type II oculocutaneous albinism. Nature 361, 72-76 (1993).

15 Sturm, R. A., Teasdale, R. D. \& Box, N. F. Human pigmentation genes: identification, structure and consequences of polymorphic variation. Gene 277, 49-62 (2001).

16 Cassidy, S. B. \& Driscoll, D. J. Prader-Willi syndrome. European J Genet 17, 3-17 (2009).

17 Gardner, J., Nakatsu, Y., Gondo, Y., Lee, S., Lyon, M., King, R. et al. The mouse pinkeyed dilution gene: association with human Prader-Willi and Angelman Syndromes. Science 257, 1121 (1992).

18 Knoll, J. H. M., Nicholls, R. D., Magenis, R. E., Glatt, K., Graham, Jr J. M., Kaplan, L. et al. Angelman syndrome: three molecular classes identified with chromosome 15q11q13-specific DNA markers. Am J Hum Genet 47, 149-155 (1990).

19 Oetting, W. S. \& King, R. A. Molecular basis of albinism: mutations and polymorphisms of pigmentation genes associated with albinism. Hum Mutat 13, 99-115 (1999).

20 Liu, F., Wollstein, A., Hysi, P. G., Ankra-Badu, G. A., Spector, T. D., Park, D. et al. Digital quantification of human eye color highlights genetic association of three new loci. PLoS Genet 6, e1000934 (2010).

21 Tully, G. Genotype versus phenotype: human pigmentation. Forensic Sci Int: Genet. 1, 105-110 (2007).

22 Valenzuela, R., Henderson, M., Walsh, M., Garrison, N., Kelch, J., Cohen-Barak, O. et al. Predicting phenotype from genotype: normal pigmentation. J Forensic Sci 55, 315-322 (2010).

23 Zaumseger, D., Rothschild, M. \& Schneider, P. SNPs for the analysis of human pigmentation genes-A comparative study. Forensic Sci Int: Genet. Supplement Series $1,544-546(2008)$

24 Duffy, D. L., Box, N. F., Chen, W., Palmer, J. S., Montgomery, G. W., James, M. R. et al. Interactive effects of MC1R and OCA2 on melanoma risk phenotypes. Hum Mol Genet 13, 447-461 (2004)

25 Jannot, A- S., Meziani, R., Bertrand, G., Gerard, B., Descamps, V., Archibaud, A. et al. Allele Variations in OCA2 gene (pink-eyed-dilution locus) are associated with genetic susceptibility to melanoma. European J Hum Genet 13, 913-920 (2005). 